\title{
Photon counting for quantum key distribution with Peltier cooled InGaAs/InP APD's.
}

\author{
Damien Stucki, Grégoire Ribordy, André Stefanov, Hugo Zbinden \\ Group of Applied Physics, University of Geneva, \\ 1211 Geneva 4, Switzerland \\ John G. Rarity, Tom Wall \\ SEE Division Defence Evaluation and Research Agency, \\ St Andrews Rd, Malvern, UK WR14 3PS
}

FINAL VERSION

(November 6, 2018)

\begin{abstract}
The performance of three types of InGaAs/InP avalanche photodiodes is investigated for photon counting at $1550 \mathrm{~nm}$ in the temperature range of thermoelectric cooling. The best one yields a dark count probability of $2.8 \cdot 10^{-5}$ per gate $(2.4 \mathrm{~ns})$ at a detection efficiency of $10 \%$ and a temperature of $-60^{\circ} \mathrm{C}$. The afterpulse probability and the timing jitter are also studied. The results obtained are compared with those of other papers and applied to the simulation of a quantum key distribution system. An error rate of $10 \%$ would be obtained after 54 kilometers.
\end{abstract}

\section{INTRODUCTION}

Quantum Key Distribution (QKD), the most advanced technology of the field of quantum information, allows two remote parties to exchange a sequence of random bits and subsequently check their secrecy [1]. It has been extensively tested in the past couple of years over distances of a few tens of kilometers [2] [6]. Its security relies on the fact that the bits are encoded on single photons.

A first consequence of this fact is that the channel transporting the photons, usually an optical fibre, must be as transparent as possible. The attenuation in silica, which is fundamentally limited by Rayleigh scattering, decreases with the wavelength. From around $2 \mathrm{~dB} / \mathrm{km}$ at $800 \mathrm{~nm}$, it goes down to $0.35 \mathrm{~dB} / \mathrm{km}$ at $1300 \mathrm{~nm}$ and even $0.2 \mathrm{~dB} / \mathrm{km}$ at $1550 \mathrm{~nm}$. Maximizing the transmission requires to work at $1550 \mathrm{~nm}$. At this wavelength, respectively $10 \%$ and $1 \%$ of photons injected in a fibre are still present after 50 and $100 \mathrm{~km}$ (as will be seen below, this value is slightly overoptimistic).

Second, single photons are difficult to detect. Although commercial detectors based on silicon avalanche photodiodes (APD) exist for wavelength below $1000 \mathrm{~nm}$, this is not true for wavelengths beyond. In order to detect single photons at $1550 \mathrm{~nm}$, commercial InGaAs/InP APD's designed for telecommunication applications have to be operated in so-called Geiger mode. In this mode, they are biased beyond the breakdown voltage. As soon as a carrier is created within the diode, it sweeps through the junction and triggers an avalanche which in turn yields a macroscopic current pulse. The carrier can be photogenerated, in which case the current pulse gives information about the arrival of a photon. It can also be generated by spurious mechanisms - like thermal excitation - which cause noise. After the detection of the avalanche, it must be quenched in order to reset the detector and prepare it for subsequent photons. Various techniques, thoroughly discussed in [7], can achieve this.

Single-photon detectors are a key component in a QKD system. They influence both the key creation rate and the error rate. Although commercial InGaAs/InP APD's have already been thoroughly tested in Geiger mode [8 11], we intend in this paper to present recent results of the characterization of such APD's and interpret them in the light of QKD. After a general introduction and the description of the set-up, we present experimental results. We look at the detection efficiency, the dark count probability, the afterpulse probability and the timing jitter. Finally, we compare these values with those presented in other works and use them in the simulation of a QKD system to estimate the possible performance.

\section{SINGLE-PHOTON DETECTION FOR QUANTUM KEY DISTRIBUTION}

Most QKD systems are synchronous, in the sense that a timing signal can be available to indicate the possible arrival of a photon. This fact makes it possible to use the APD's in gated mode, where a biasing pulse is applied by a suitable generator to gate the detector on. We only consider this mode which is well suited to QKD. 
An ideal single photon detector should produce an electronic logical signal when and only when a photon strikes it. Real detectors unfortunately differ from this simple picture. First, the detector sometime fails to record a photon. The probability for an impinging photon to be detected - also called detection efficiency - is lower than $100 \%$. Second, the detector also has a non-zero probability to produce a count even though no photon is present. Such an event can stem from the thermal generation of a carrier in the sensitive area. In this case, it is known as a dark count. It can also arise from the release of a charge trapped in the junction in the course of a previous avalanche, in which case it is called an afterpulse. Finally, the time between the absorption of a photon, the triggering of the avalanche and its detection will be statistically distributed around an average value. This uncertainty is called the timing jitter.

In order to work well for QKD, a single-photon detector should have a reasonably high detection efficiency, and low dark count and afterpulse probabilities. Although it is not very critical, there are also constraints on the timing jitter. A given QKD system can be characterized by its raw bit rate $R$ and its quantum bit error rate $Q B E R$. The bit rate is basically equal to the product of the probability $p_{T}$ of a photon to reach the receiver's detector, the detection efficiency $\eta_{\text {det }}$, and the repetition frequency $f_{\text {rep }}$.

$$
R=p_{T} \cdot \eta_{\text {det }} \cdot f_{\text {rep }}
$$

The QBER is equal to the ratio of erroneous counts over total counts. We will consider only errors caused by the detectors, which constitute the dominant contribution. It can be written in terms of probabilities and is essentially equal to the ratio of the probability to record a false count - the sum of the dark count probability $\left(p_{d c}\right)$ and afterpulse

probability $\left(\left(p_{T} \cdot \eta_{\text {det }}+p_{d c}\right) \cdot \sum_{n=1}^{\infty} p_{a p}\left(\Delta t_{n}\right)\right)$ - over that of recording a correct one. Note that $\Delta t_{n}=n \cdot \frac{1}{f_{\text {rep }}}$ and $p_{a p}(\Delta t)$ is the probability to register an afterpulse during a gate arriving $\Delta t$ after one that yielded an avalanche. In the low dark count limit $\left(p_{d c} \ll 1\right)$ we can ignore afterpulsing from dark counts and

$$
Q B E R=\frac{\text { incorrect counts }}{\text { total counts }} \approx \frac{p_{d c}+p_{T} \cdot \eta_{d e t} \cdot \sum_{n=1}^{\infty} p_{a p}\left(\Delta t_{n}\right)}{p_{T} \cdot \eta_{\text {det }}}=\frac{p_{d c}}{p_{T} \cdot \eta_{\text {det }}}+\sum_{n=1}^{\infty} p_{a p}\left(\Delta t_{n}\right)
$$

Without going too much into details (see [1] for a discussion of these quantities), a system should yield a $R$ as high as possible and a $Q B E R$ as low as possible. A low detection efficiency causes both a reduction of $R$ and an increase of $Q B E R$. High dark count and afterpulse probabilities yield high $Q B E R$. The dark count probability can nevertheless be reduced by cooling the detector. Even though cryogenic temperatures are easy to obtain in the laboratory, for example by using liquid nitrogen, they are not suited to the realization of a commercial prototype. We only consider here the temperature range that is easily attainable with thermoelectric cooling (down to $-60^{\circ} \mathrm{C}$ ). The dark count probability can also be reduced by recording counts in a small time window around the arrival of the photons. The width of this window is ultimately limited by the timing jitter.

\section{DETECTION EFFICIENCY AND DARK COUNTS}

Three different types of InGaAs/InP APD's (Epitaxx EPM 239 AA, EG\&G 30733, and NEC NDL 5551) were tested and compared. Although the results presented in this paper were obtained with one single Epitaxx APD, additional tests performed on other specimens of this APD and not reported here yielded similar results in photon counting mode. As for the EG\&G and NEC APD's, only one APD of each was tested. Thus we cannot present information about differences among samples. Both the Epitaxx and the NEC APD's were mounted on a copper block equipped with a heating coil. It was placed in a closed tube and immersed in liquid nitrogen. The temperature was then adjusted by heating the arrangement. This solution was selected because of the availability of the equipment, but a Peltier cooler would have been just as suitable for the target temperature range. The EG\&G APD is mounted in a DIL package and includes a Peltier cooler and thermistor. The whole package was then mounted on a single-stage peltier cooler in an insulated metallic box. The hot side of the thermoelectric element was placed on a radiator equipped with a fan.

The schematic set-up for measuring the quantum detection efficiency and the dark count probability of the APD's is shown in Fig. 1. A delay generator (Stanford Research Systems DG 535) acts as the time base of the system and generates signals, unless otherwise mentioned, at a frequency of $10 \mathrm{kHz}$. It triggers first a pigtailed semiconductor laser (MRV MRLD14CD5015), which generates short light pulses $(\simeq 350 \mathrm{ps})$ at $1550 \mathrm{~nm}$. These pulses are attenuated to the single photon level by a calibrated variable attenuator (EXFO IQ-3100, maximum attenuation $>100 \mathrm{~dB}$ ) equipped with FC/APC connectors. The output of this attenuator is connected to the pigtail of the APD. The attenuator has a shutter that can be used to turn on and off the illumination of the detector. 
The delay generator also triggers a gate generator that produces square biasing pulses with an amplitude of 6.5 $\mathrm{V}$ and a full width at half maximum of $2.4 \mathrm{~ns}$ (see Fig. 2). The delays are set so that the photons impinge on the APD's sensitive area when it is gated on. This pulse is superimposed upon an offset voltage, whose level is adjusted to control the excess bias voltage. The avalanche signal is then detected with a discriminator which generates logical output pulses. These are finally registered with a counter. As can be seen in Fig. 2, the rising and falling edges of the gate pulse are sharp. They produce transients in the output signal that can be larger than the avalanche itself, especially for low excess bias voltages. The output of the discriminator is fed into a coincidence circuit in order to reject the transients and record only the avalanche signals.

For a given bias voltage, we measured first the dark count probability. The shutter was closed, and counts accumulated. We selected the integration time, to keep the statistical uncertainty typically around $5 \%$, except for the lowest bias voltage. In this case, the low dark count probability prevented us from going below 10\% (accumulation of at least 100 counts). The dark count probability is then simply calculated as the ratio of the number of counts over the product of the repetition frequency and integration time. In order to obtain the detection efficiency, we opened the shutter and registered counts for a given time. It was then estimated by subtracting the dark count probability. The Poissonian statistics of the number of photons per pulse was taken into account. We then changed the excess bias voltage and repeated the measurements.

The results for the three APD's are shown in Fig. 3, where the dark count probability is plotted as a function of the detection efficiency. The figure shows the error bars stemming from statistical uncertainties in the case of the Epitaxx-APD for the detection efficiency. Those concerning the dark count probability are not shown since they are smaller than the symbols. For the other APD's, the uncertainties are similar but were omitted for the sake of clarity. Considering first the data corresponding to the Epitaxx APD at $-40^{\circ} \mathrm{C}$ and $-60^{\circ} \mathrm{C}$, we notice a positive correlation between these two quantities. The points correspond to different excess bias voltage. Both the detection efficiency and the dark count probability increase with excess bias voltage. This reflects the fact that a strong applied field enhances the probability for a carrier to start an avalanche by impact ionization. The data can be well approximated by an exponential fit. The slope is identical for both $-40^{\circ} \mathrm{C}$ and $-60^{\circ} \mathrm{C}$. The results also show that the dark count probability can be reduced by cooling the detector. When the temperature is reduced, the curves shift downwards. At a detection efficiency of approximately $10 \%$, the dark count probability is equal to $6 \cdot 10^{-5}$ and $2.8 \cdot 10^{-5}$ at $-40^{\circ} \mathrm{C}$ and $-60^{\circ} \mathrm{C}$ respectively.

We verified that the contribution of afterpulses to the measured data is negligible. The repetition frequency is indeed so low that the traps filled by an avalanche have already been emptied when the next gate pulse is applied. To establish this, we changed the repetition frequency and verified that the dark count probability did not change. At the maximum repetition frequency of $800 \mathrm{kHz}$ and $\mathrm{T}=-40^{\circ} \mathrm{C}$, we did not measure any increase. In the next, section we will discuss the issue of afterpulses in more detail.

The NEC-APD and the EG\&G-APD show similar performance (see also Fig. 3), but are not as good as the Epitaxx-APD. Their dark count probability is around $3.5 \cdot 10^{-4}$ at an efficiency of $10 \%\left(\mathrm{~T}=-60^{\circ} \mathrm{C}\right)$, while it is as low as $2.8 \cdot 10^{-5}$, more than one order of magnitude smaller, for the Epitaxx-APD in similar conditions.

It is clear that for applications where the dark count probability is an important issue, cooling the detector has a significant impact. Since it is critical for QKD, one could wonder why we did not test the detectors to temperatures lower than $-60^{\circ} \mathrm{C}$. The first reason is that we want to be able to cool the detectors with thermoelectric elements (Peltier coolers). They are indeed practical and reliable. One could nevertheless object that other proven technologies (e.g. Stirling cycle coolers) that allow to reach lower temperatures exist. This would however not help us. More importantly, when the temperature is lowered, the breakdown voltage $V_{B}$ decreases. As explained by Cova $[8]$ as well as Hiskett [11], $V_{B}$ should always remain larger than the so called reach-through voltage $V_{R T}$ if one wants to have the carrier generated in the absorption layer injected in the high-field multiplication zone. As $V_{R T}$ does not change with temperature, contrary to $V_{B}$, the APD might breakdown at a voltage smaller than the reachthrough level below a certain temperature. The Epitaxx-APD for example did not operate below $-90^{\circ} \mathrm{C}$. Finally, when cooling the detectors, the lifetime of the trapped charges, that cause afterpulses, increases.

\section{AFTERPULSES}

We investigate now the afterpulse probability at different temperatures and for the various APD's. The set-up is similar to the one used in the previous section (see Fig. 1). A third timing signal provided by the delay generator is combined through an OR-gate to the one sent to the gate generator. The APD is thus turned on at two subsequent times separated by a variable delay $\Delta t$. The laser pulse is still synchronized with the first gate. When disconnecting the OR-gate input corresponding to the second gate pulse, one is back in the situation presented in the previous section. This arrangement was used to measure the detection efficiency and dark count probability, and verify that 
the APD was operating properly. The results presented below were all obtained with a bias voltage corresponding to a detection efficiency of approximately $10 \%$. With both input of the OR-gate connected, one could count events happening both in the first and second gate pulse. In order to register only the events where a count happened both in the first and the second window, we used a coincidence circuit. One can then use these data to evaluate the probability to register a pulse in the second gate knowing that one was obtained in the first one for various time delays between both gates. After subtracting the dark count probability, one obtains the afterpulse probability $p_{a p}(\Delta t)$.

The results for the Epitaxx-APD are shown in Fig. 4. The probability $p_{a p}$ is plotted versus the time interval $\Delta t$ between the two gates, with $\Delta t$ up to $10 \mu \mathrm{s}$. It starts at approximately $10^{-2}$ and decreases. One would expect that it would go down to zero for a time long enough. The uncertainty nevertheless increases with $\Delta t$, since the number of counts accumulated becomes smaller. In addition, one expects $p_{a p}$ to become larger, for a given value of $\Delta t$, when the temperature is reduced. The lifetime of the traps should indeed become longer. While this seems to be true for times beyond $5 \mu s$, it is not before. Cova deduced from measurements with Si-APD's that several trapping levels, with different time constants, contribute to afterpulses [12]. This is likely to remain true with InGaAs/InP APD's. A different temperature dependence of the lifetime for the trapping levels might explain this result. Figure 4 also suggest that temperature may not have a strong impact on the afterpulse probability in the Peltier cooling range.

When considering the other APD's, one finds that their afterpulse probability is both lower than that of the EpitaxxAPD and decreases faster (Fig. 5). Differences among the different APD's is not surprising, since the trap density depends intrinsically on the structural quality of a diode. It is reasonable to conjecture that improvements could be obtained with appropriate efforts by manufacturers.

These results also demonstrate that the afterpulse probability after $100 \mu$ s is very low (not shown on Fig. 4). When using a repetition frequency of $10 \mathrm{kHz}$, the trapped charges are always released between two gates. This justifies our claim that we were able to isolate dark counts effects from afterpulses in the measurements presented in the previous section.

\section{A. Avalanche pulse analysis}

As explained by Cova and his coworkers in [8] and [13], it is possible to obtain valuable information about the trapping phenomena in an APD by analyzing the avalanche pulse. To do that, we had to modify slightly the experimental set-up. It is indeed necessary to apply long gate pulses $\left(t_{\text {Gate }} \geq 20 \mathrm{~ns}\right)$. Since the width of the gate produced by our generator cannot be increased, we directly fed the variable output of the delay generator into a custom made amplifier (maximum amplitude $8 \mathrm{~V}$ ). We then recorded the avalanche pulses with a digital oscilloscope (Tektronix TDS 580C, $1 \mathrm{GHz}$ bandwidth). The APD's output could also be fed into a time-to-digital (TDC) converter to record counts occurring within a precisely defined time window. We used this possibility to accumulate counts over a 2 ns long region of the gates, yielding results that can be compared with those obtained with short gates. The rest of the set-up was identical.

When looking at a typical trace (Fig. 6), one notices the avalanche pulse and the transient corresponding to the falling edge of the gate pulse. The avalanche increases sharply and reaches a maximum after $2.5 \mathrm{~ns}$, before reaching a regime where it remains constant for about $5 \mathrm{~ns}$. Then, it decreases to a plateau that lasts until the end of the gate. This decrease indicates a change in the breakdown voltage of the junction during the avalanche. They suggest two mechanisms for this effect. First, the fact that traps get filled during an avalanche pulse changes the charge distribution in the junction and increases $V_{B}$. The typical time constant of this phenomenon is of the order of a few nanosecond. The second effect is that the current flowing in the junction raises its temperature, which also increases $V_{B}$. In this case the time constant is larger, typically a few hundred nanoseconds. In order to observe it, even longer gate pulses are necessary (results not shown here). Both of these phenomenon yield in turn a reduction of the excess bias applied on the junction, and a decrease of the pulse amplitude. Information about the diode structure (dopant concentration), as well as the peak to plateau difference can be used to estimate the fraction of filled traps. However this structural information was not available. Nevertheless we can conclude from this avalanche pulse analysis that the trapping effects are significant in the Epitaxx-APD's. In addition, their filling takes about $5-7$ nanoseconds at a gate voltage corresponding to an efficiency of about $10 \%$. This indicates that it is essential for the duration of the gate pulse to be smaller than this time, if one wants to reduce the afterpulse probability by a limitation of the charge transit in the junction. When measuring quantitatively the afterpulse probability with long gates (Fig. 7), we indeed observed results approximately two orders of magnitude higher than those obtained with short gates. After $100 \mathrm{~ns}$, the afterpulse probability is close to $100 \%$ when long gates are used, while it is only of the order of $1 \%$ with short gates. The fact that avalanches are not quenched before the end of the gate, lasting $20 \mathrm{~ns}$, explains this result. Note that in the case of long gates, the afterpulses are accumulated in a $2 \mathrm{~ns}$ window defined with the TDC and not over the entire gate duration. This guarantees that the results obtained with the two types of gates can be compared. 


\section{B. Gate amplitude}

Contrary to the short gate generator, the long gate generator allows to change the gate amplitude and to investigate its effect on the performance of the detector. Cova and his coworkers suggest that holding the detector biased close to the breakdown voltage during the off-periods enhances detrapping through the Franz-Keldish effect (see [8]). To verify this, we used gate amplitudes of 3, 4, 6 and $8 \mathrm{~V}$. In each case, we made sure that the excess bias voltage was such that the detection efficiency was about $10 \%$ and measured the afterpulse probability as discussed above. The results are shown in Fig. 7. It appears clearly that, contrary to what we expected, a higher gate amplitude, which is corollary to holding the detector well below the breakdown voltage during the off-periods, reduces the time constant of the afterpulse probability. It indicates that, in III-V hetero-junctions such as those of InGaAs/InP APD's, other phenomena dominate the Franz-Keldish effect. Further research is necessary to better understand them.

We also measured the dark count probability as a function of the quantum detection efficiency for the various gates amplitudes with a repetition frequency of $10 \mathrm{kHz}$ (Fig. 8). The temperature was $-50^{\circ} \mathrm{C}$. The results clearly indicate that the performance of the detectors is identical in all cases. The amplitude of the gate influences thus only the afterpulse probability. With long gates (20 ns) nevertheless a dark count probability of $10^{-4}$ for a detection efficiency of $10 \%$ was measured. This performance is not as good as that obtained with short gates. This can be explained by the higher afterpulse probability. In this case, the repetition frequency was not low enough to allow the traps to empty themselves.

In summary, if one wants to reduce the afterpulse probability when working with InGaAs APD's, the gates should be kept as short as possible and their amplitude as high as possible.

\section{TIMING JITTER}

The timing jitter is the last property of the APD's that we investigated. We used the same set-up as above with long gates and fed the output of the detectors into the TDC. When sending light pulses in coincidence with the gate, we obtained time spectra consisting of a broad pedestal corresponding to dark counts and a well defined peak corresponding to the light pulse. We can deduce from the measured full width at half maximum (FWHM) of the peak and that of the laser pulse $(\simeq 350 \mathrm{ps})$ the timing jitter of the APD. Figure 9 shows the results obtained at three different temperatures and for the Epitaxx-APD as a function of the detection efficiency. One can see that the values typically range between 500 and 300 ps. The jitter decreases with the efficiency. At $10 \%$ its value is around 450 . Finally, the temperature, in the studied range, does not have a significant impact.

\section{COMPARISON WITH PREVIOUS RESULTS}

Let us now compare the results obtained for the Epitaxx-APD with those presented by Hiskett and his coworkers 111 for the detection of photons at 1550nm. They tested two different types of Fujitsu-APD's (30FPD13U81SR with $80 \mu \mathrm{m}$ active area diameter, and FPD13W31RT with a $30 \mu \mathrm{m}$ active area diameter). When cooled to $-196^{\circ} \mathrm{C}$, the first device yields a dark count probability of $10^{-4}$ in a 2.5 ns window for a detection efficiency of $10 \%$. As a reminder, we obtained for the Epitaxx-APD a dark count probability of $2.8 \cdot 10^{-5}$ at $-60^{\circ} \mathrm{C}$ and $\eta_{\text {det }}=10 \%$. In spite of the fact that they do not measure the afterpulse probability, they observe that for their APD cooled to $-196^{\circ} \mathrm{C}$, the dark count probability already doubles when the repetition frequency is increased to $30 \mathrm{kHz}$. Both the higher dark count and afterpulse probability would yield lower performance in a QKD system with respect to those that would be obtained with an Epitaxx-APD.

In addition, the APD's tested in [9] (Fujitsu FPD5W1KS) yield similar performance as those presented by Hiskett $\left(P_{d c}=10^{-4}\right.$ at $\left.\eta_{d e t}=10 \%\right)$. These results are however obtained at a temperature of $-80^{\circ} \mathrm{C}$, where the APD's performance is optimal.

Finally, the EG\&G and NEC APD's had been previously tested using a passive quenching approach [10]. They showed a higher dark count probability $\left(p_{d c} \approx 2.5 \cdot 10^{-3}\right.$ per 2.4 ns with $\left.\eta_{\text {det }}=10 \%\right)$. A significant number of dark counts arose due to afterpulsing from dark counts alone. This highlights the advantage of gated operation where the only significant afterpulsing contribution comes from the light counts. Afterpulsing was measured using a correlation technique and integrated over long times so that it cannot be directly compared but appears to be of a similar magnitude. When correcting the dark count probability for afterpulsing, a value only slightly higher than in gated mode $\left(\geq 5 \cdot 10^{-4}\right)$ was found. 


\section{APPLICATION TO QKD}

Let us now use the results obtained here, to simulate QKD. We will first neglect the effect of afterpulses. We will also consider that the detectors are the only source of errors. The raw key creation rate can be calculated using Equation (1). It is necessary however to consider $p_{T}$, the probability for a photon to reach the receiver's detectors, in more details. Let us rewrite this quantity as

$$
p_{T}=\mu \cdot T_{L} \cdot T_{R}
$$

In this equation, $\mu$ represents the probability for an emitted pulse to contain at least one photon. Since ideal single photon sources do not exist, one approximates them with attenuated laser pulses containing an average number of photons of typically 0.1 . Here, we will follow this convention and set $\mu=0.1$. The second quantity, $T_{L}$, represents the transmission probability of the a photon through the optical fibre line between the emitter and the receiver. We will assume that the attenuation at $1550 \mathrm{~nm}$ in optical fibres is $0.25 \mathrm{~dB} / \mathrm{km}$, somehow higher than the lowest attenuation measured. Installed fibres tend indeed to show higher attenuation than the absolute minimum and feature splices, typically every few kilometers. Finally $T_{R}$ represents the transmission of the receiver system. We will set it to 0.5 , corresponding to an attenuation of $3 \mathrm{~dB}$.

Setting $\eta_{d e t}=10 \%$, as we experimentally obtained, one can calculate $R / f_{\text {rep }}$ as a function of the distance in kilometers (Fig. 10). Even when the distance is equal to zero, the normalized raw rate is smaller than unity, because most pulses are empty, the detection efficiency is low and the transmission of the receiver not perfect. The normalized rate then decreases with the distance. Note that the sifted key rate, after basis reconciliation, is half the raw rate plotted on Fig. 10.

We also plotted on Fig. 10 the $Q B E R$ calculated with Equ. 2 for the Epitaxx-APD at $-40^{\circ} \mathrm{C}$ and $-60^{\circ} \mathrm{C}$, for the EG\&G or NEC APD's at $-60^{\circ} \mathrm{C}$, and finally for a hypothetical improved detector featuring a dark count probability ten times smaller than that of the Epitaxx-APD. The impact of this quantity appears clearly. Although the EpitaxxAPD yields satisfactory results $(Q B E R=10 \%$ after $54 \mathrm{~km})$, the EG\&G or NEC APD's are not suitable for QKD. Finally, the hypothetical detector would allow QKD over distances well beyond $50 \mathrm{~km}$. Note that the fraction of bits lost during key distillation (error correction and privacy amplification) can be estimated to $50 \%$ and $85 \%$ at a $Q B E R$ of $5 \%$ and $10 \%$ respectively. The error rate should thus clearly be kept below $10 \%$.

These results were obtained assuming that afterpulses could be neglected. This is true as long as the repetition frequency is slow enough. Looking at Fig. 10 again, it appears clearly that the normalized raw rate decreases steeply with distance. At $70 \mathrm{~km}$ for example, it is only $10^{-4}$ of the repetition rate. In order to generate a non-negligible bit stream, a high repetition frequency is necessary. The question now is to evaluate the impact of afterpulses under these conditions.

Let us assume that we will tolerate an increase of the $Q B E R$ of $1 \%$, which implies that $\sum_{n=1}^{\infty} p_{a p}\left(\Delta t_{n}=n \cdot 1 / f_{r e p}\right)$ must be smaller than $10^{-2}$. Whether this condition is fulfilled depends on the time dependence of $p_{a p}$ and the repetition frequency. When considering the Epitaxx-APD with short gates at $-60^{\circ} \mathrm{C}$ and a repetition frequency of for example $1 \mathrm{MHz}$, one finds that the cumulated afterpulse probability is equal to $1.4 \%$. Note that the values $p_{a p}(\Delta t)$ were obtained from an exponential decay fit of the data shown on Fig. 4 and consisting of three terms. This probability can be reduced by suppressing an appropriate number of gates after each recorded detection, in order to wait for the trapping levels to empty. In this example, such a hold-off mechanism must skip two gates after each detection to bring the cumulated probability below $1 \%$, while the key creation rate would be affected in a negligible way. For higher repetition frequencies, the number of skipped gates must be increased (14 gates at $2 \mathrm{MHz}$ ). Finally, this analysis also indicates that long gates are not suitable for QKD at a high repetition frequency because of the higher afterpulse probability they induce.

Let us finally discuss the timing jitter of the detectors in the context of QKD. This quantity is important for two reasons. First, it is possible to reduce dark counts by time discrimination. Second, certain QKD set-ups involve interferometers where different optical paths must be distinguished in the time domain. These must clearly induce time differences in the arrival of the photons larger than the jitter. Since interferometers with large path differences are in general difficult to stabilize, a small jitter constitutes an advantage. In order to keep the overlap of two gaussians smaller than $5 \%$ with a jitter of $450 \mathrm{ps}$, one must select a time difference of at least $2.6 \mathrm{~ns}$, corresponding in optical fibres to a length of $0.52 \mathrm{~m}$ approximately.

\section{CONCLUSION}

In this paper, we have tested three types of InGaAs/InP APD's in the temperature range of Peltier cooling at 1550 $\mathrm{nm}$, in the light of QKD. We have first investigated their detection efficiency and dark count probability. The best 
APD - the Epitaxx-APD - yielded dark count probabilities of $2.8 \cdot 10^{-5}$ and $6 \cdot 10^{-5}$ for temperatures of $-60^{\circ} \mathrm{C}$ and $-40^{\circ} \mathrm{C}$ respectively and an efficiency of $10 \%$. We also measured the afterpulse probability. The importance of limiting the duration of the avalanche - and use short gates - to reduce this quantity was clearly demonstrated. The timing jitter of the Epitaxx-APD was also investigated.

We compared the results obtained with those published in other papers on InGaAs/InP APD's. The performance of the Epitaxx-APD constitutes the best one reported to date for photon counting at $1550 \mathrm{~nm}$. In addition, it is obtained at a temperature easily reachable with Peltier cooling, whereas other diodes required more sophisticated cooling.

These results were then applied to the simulation of a QKD system, illustrating that among the three types of APD's, the Epitaxx-APD is the only one to yield satisfactory performance. Error rates of $5 \%$ and $10 \%$ would be obtained after respectively $40 \mathrm{~km}$ and $54 \mathrm{~km}$. Assuming a repetition frequency of $1 \mathrm{MHz}$, the raw key creation rate would be in the $100 \mathrm{~Hz}$ range. Finally, they indicate the necessity to use a hold-off mechanism when operating these APD's at a high repetition frequency.

Acknowledgement 1 The authors would like to thank Prof. Sergio Cova for helpful discussions. This work was supported by the Swiss OFES and the European EQCSPOT project.

\section{FIGURE CAPTIONS}

Figure 1

Experimental setup.

Figure 2

Gate pulse produced by the short gate generator.

Figure 3

Dark count probability per gate pulse of 2.4 ns versus detection efficiency.

Figure 4

Afterpulse probability per gate pulse of 2.4 ns versus time since previous avalanche.

Figure 5

Afterpulse probability per gate pulse of 2.4 ns versus time since previous avalanche.

Figure 6

Avalanche pulse of Epitaxx avalanche photodiode.

Figure 7

Afterpulse probability per $2 \mathrm{~ns}$ versus time since previous avalanche for long gates $(20 \mathrm{~ns})$ of $3,4,6$ and $8 \mathrm{~V}$ amplitude.

Figure 8

Dark count probability per 2 ns versus detection efficiency for long gates (20 ns) of 3, 4, 6 and $8 \mathrm{~V}$ amplitude.

Figure 9

Timing jitter (full width at half maximum) versus detection efficiency.

Figure 10

QBER (solid lines) and normalized raw key creation rate (dashed line) versus distance for Epitaxx APD at two temperatures, EG\&G and NEC APD's, and a hypothetical photodiode with a dark count probability reduced by a factor of ten $\left(2.8 \cdot 10^{-6}\right)$ with respect to that of the Epitaxx APD. 
[1] Gisin, N., Ribordy, G., Tittel, W., and Zbinden, H., 2000, Quantum Cryptography, submitted to Reviews of Modern Physics, quant-ph/0101098.

[2] Townsend, P. D., 1998, Quantum cryptography on optical fiber networks, Optical Fiber Technology, 4, 345-370.

[3] Bourennane, M., Gibson, F., Karlsson, A., Hening, A., Jonsson, P., Tsegaye, T., Ljunggren, D., and Sundberg, E., 1999, Experiments on long wavelength (1550nm) "plug and play" quantum cryptography systems, Optics Express, 4, 383-387.

[4] Ribordy, G., Gautier, J.-D., Gisin, N., Guinnard, O., and Zbinden, H., 2000, Fast and user-friendly quantum key distribution, Journal of Modern Optics, 47, 517-531.

[5] Hughes, R., Morgan, G., Peterson, C., 2000, Quantum key distribution over a 48km optical fibre network, Journal of Modern Optics, 47, 533-547.

[6] Bethune, D., Risk, W., 2000, An Autocompensating Fiber-Optic Quantum Cryptography System Based on Polarization Splittinh of Light, IEEE Journal of Quantum Electronics, 36, 340-347.

[7] Cova, S., Ghioni, M., Lacaita, A., Smori, C. and Zappa, F., 1996, Avalanche photodiodes and quenching circuits for single-photon detection, Applied Optics, 35, 1956-1976.

[8] Lacaita, A., Zappa, F., Cova, S., and Lovati, P., 1996, Single-photon detection beyond $1 \mu$ m: performance of commercially available InGaAs/InP detectors, Applied Optics, 35, 2986-2996.

[9] Ribordy, G., Gautier, J.-D., Zbinden, H., and Gisin, N., 1998, Performance of InGaAs/InP avalanche photodiodes as gated-mode photon counters, Applied Optics, 37, 2272-2277.

[10] Rarity, J., Wall, T., Ridley, K., Owens, P., and Tapster, P., 2000, single-Photon Counting for the 1300-1600-nm Range by Use of Peltier-Cooled and Passively Quenched InGaAs Avalanche Photodiodes", Applied Optics, 39, 6746-6753.

[11] Hiskett, P., Buller, G., Loudon, A., smith, J., Gontijo, I., Walker, A., Townsend, P., and Robertson, M., 2000, Performance and Desing of InGaAs/InP Photodiodes for Single-Photon Counting at $1.55 \mu \mathrm{m}$, Applied Optics, 39, 6818-6829.

[12] Cova, S., and Lacaita, A., 1991 Trapping Phenomena in Avalanche Photodiodes on Nanosecond Scale, IEEE Electron Device Letters, 12, 685-687.

[13] Lacaita, A., Francese, P., Zappa, F., and Cova, S., 1994, Single-photon detection beyond $1 \mu$ m: performance of commercially available germanium photodiodes, Applied Optics, 30, 6902-6918. 


\section{Figure 1}

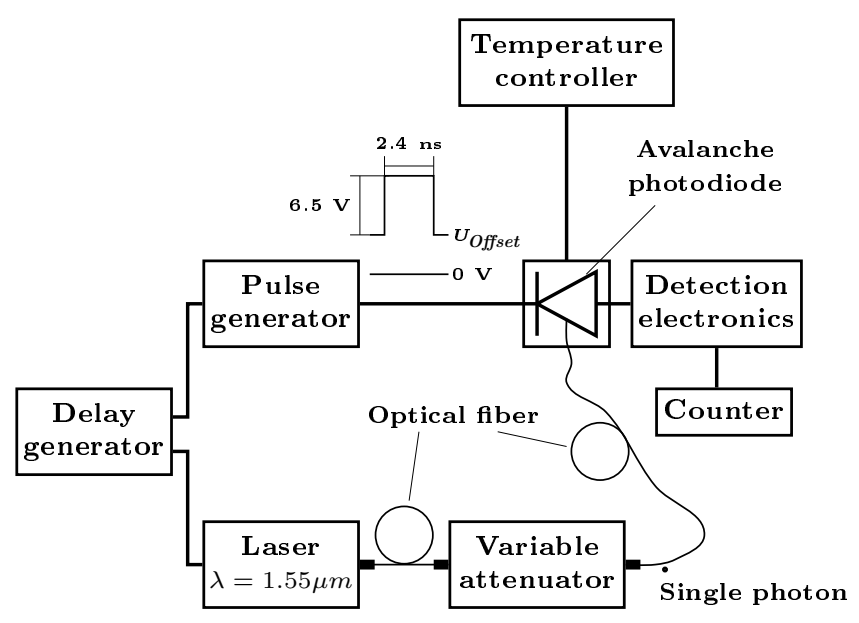


Figure 2

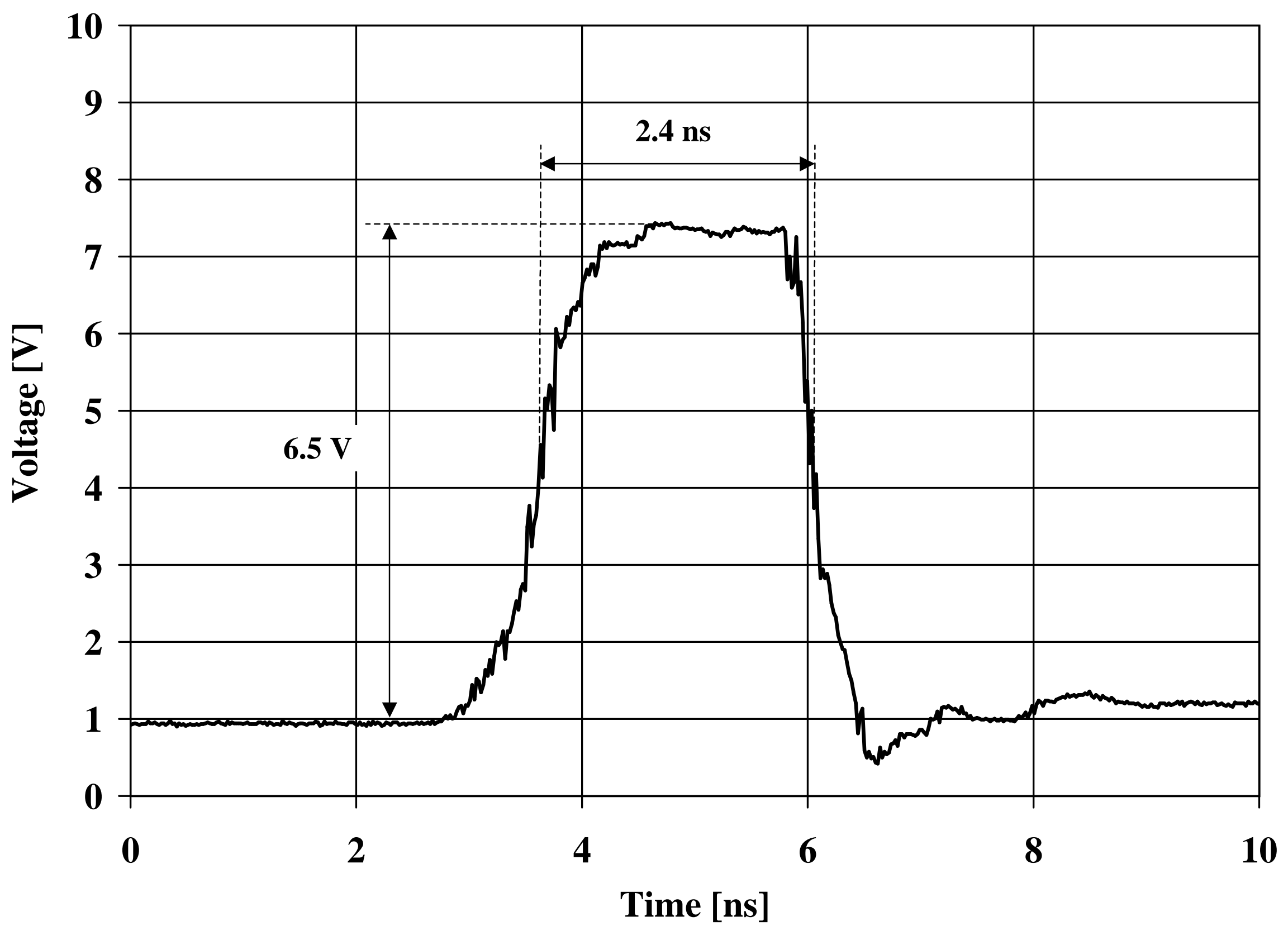


Figure 3

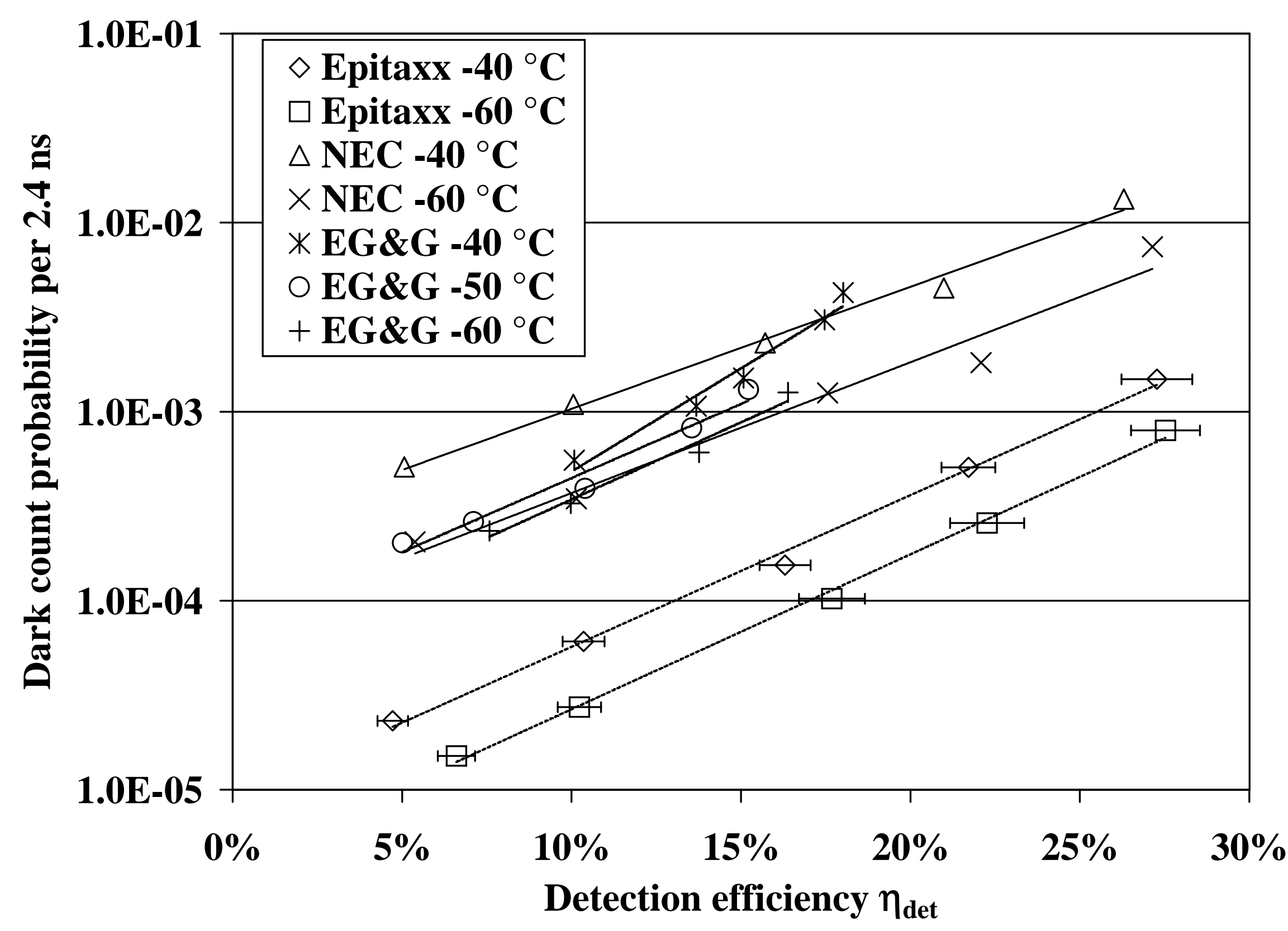


Figure 4

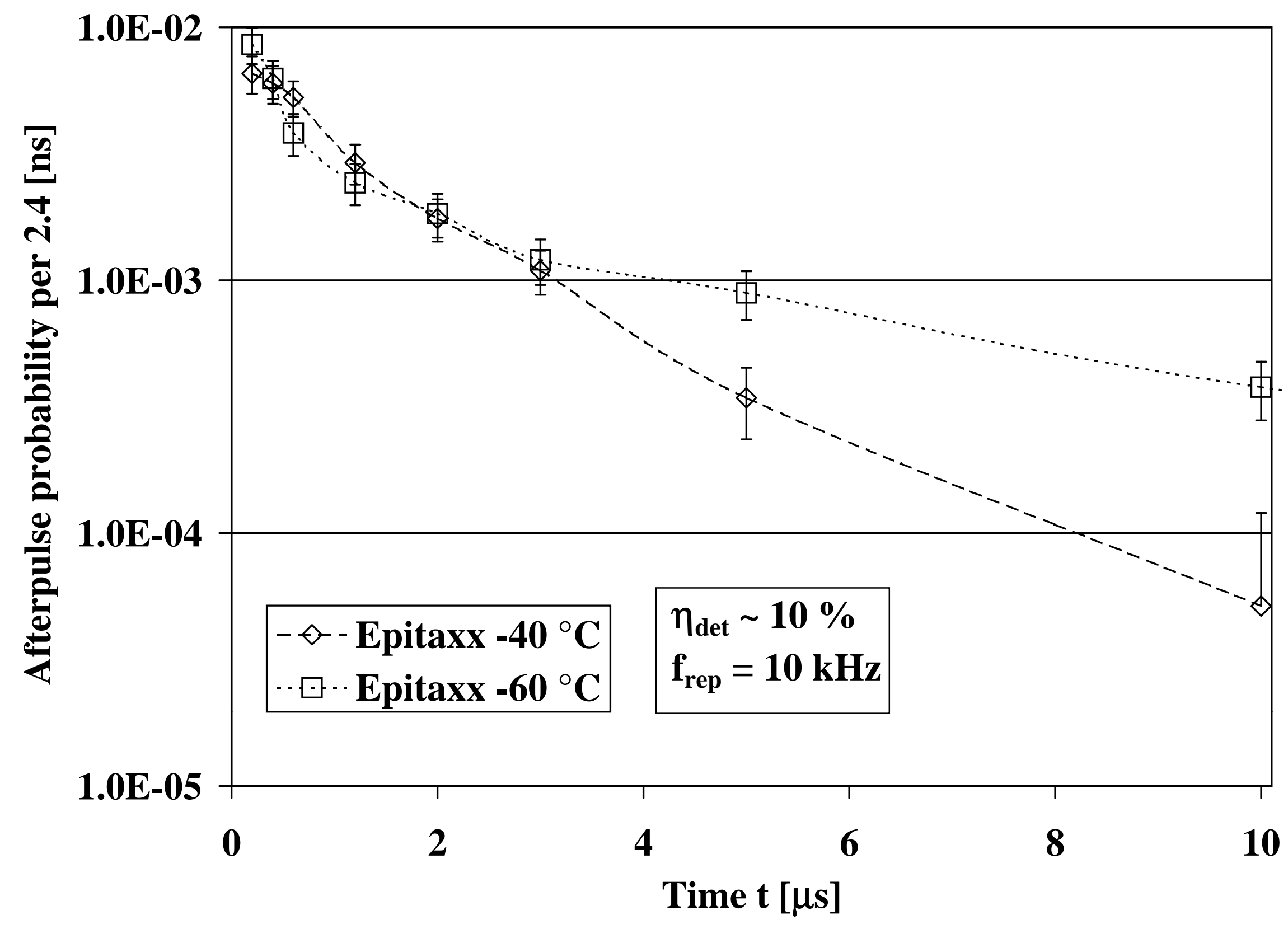


Figure 5

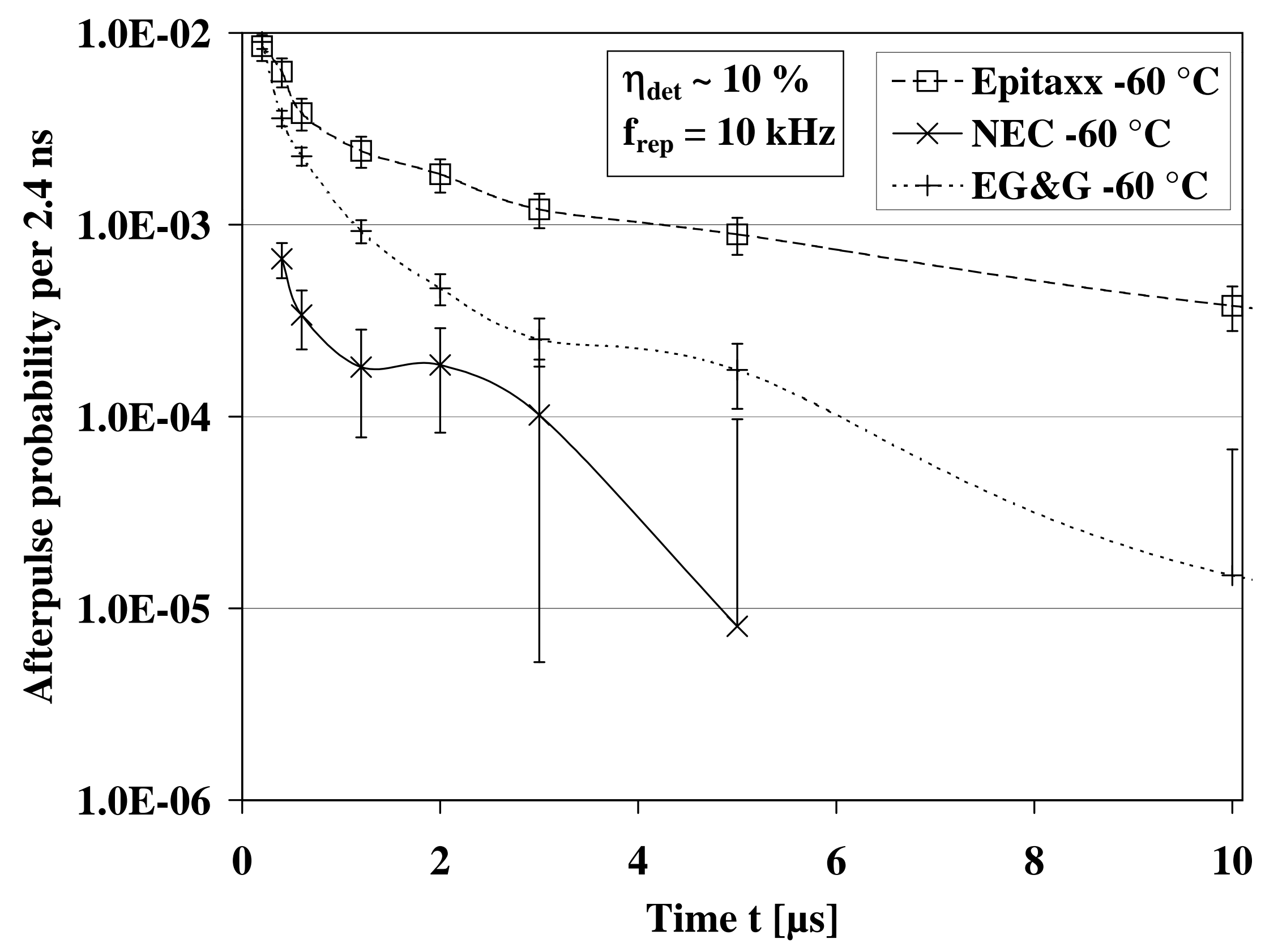


Figure 6

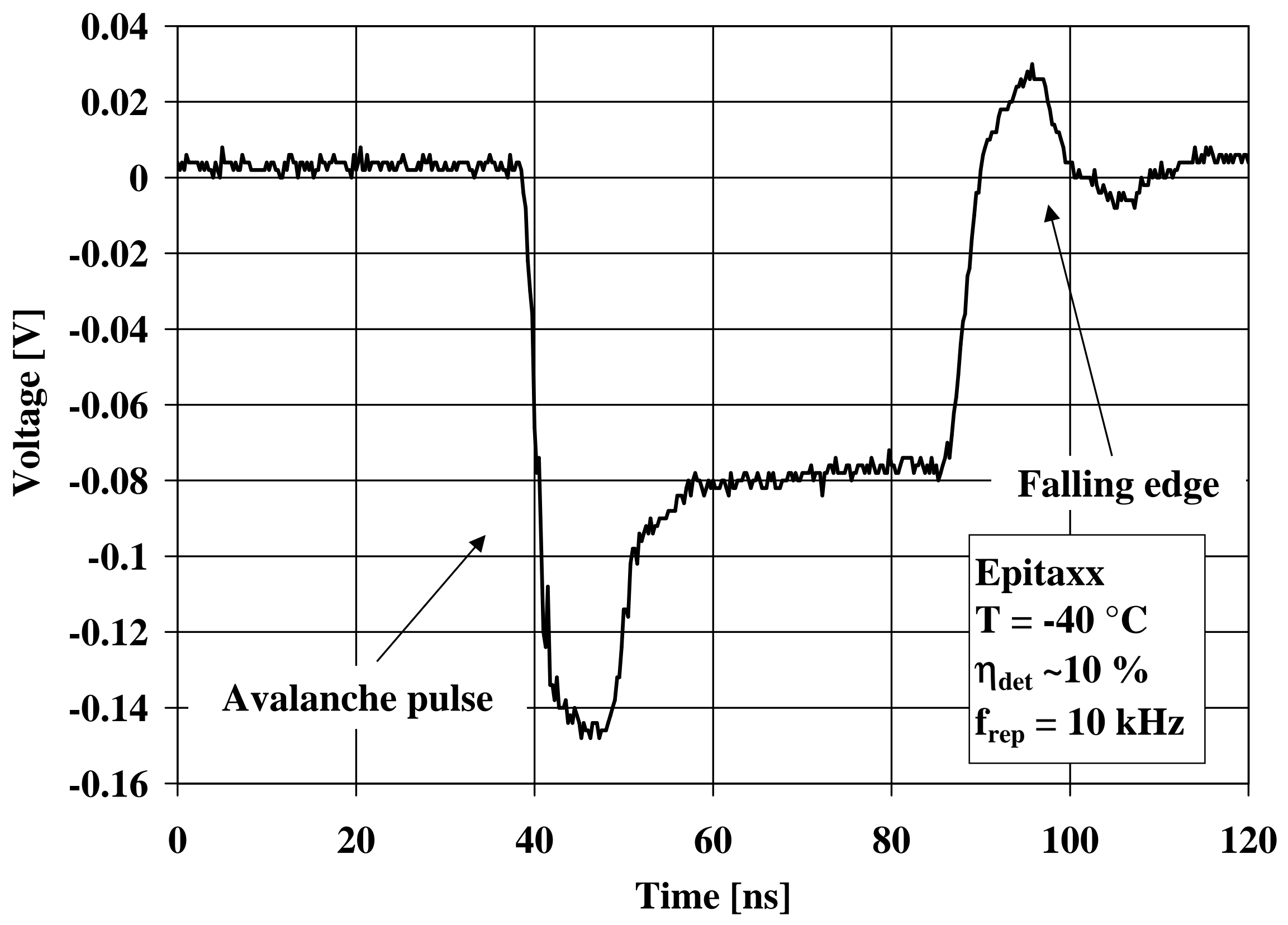


Figure 7

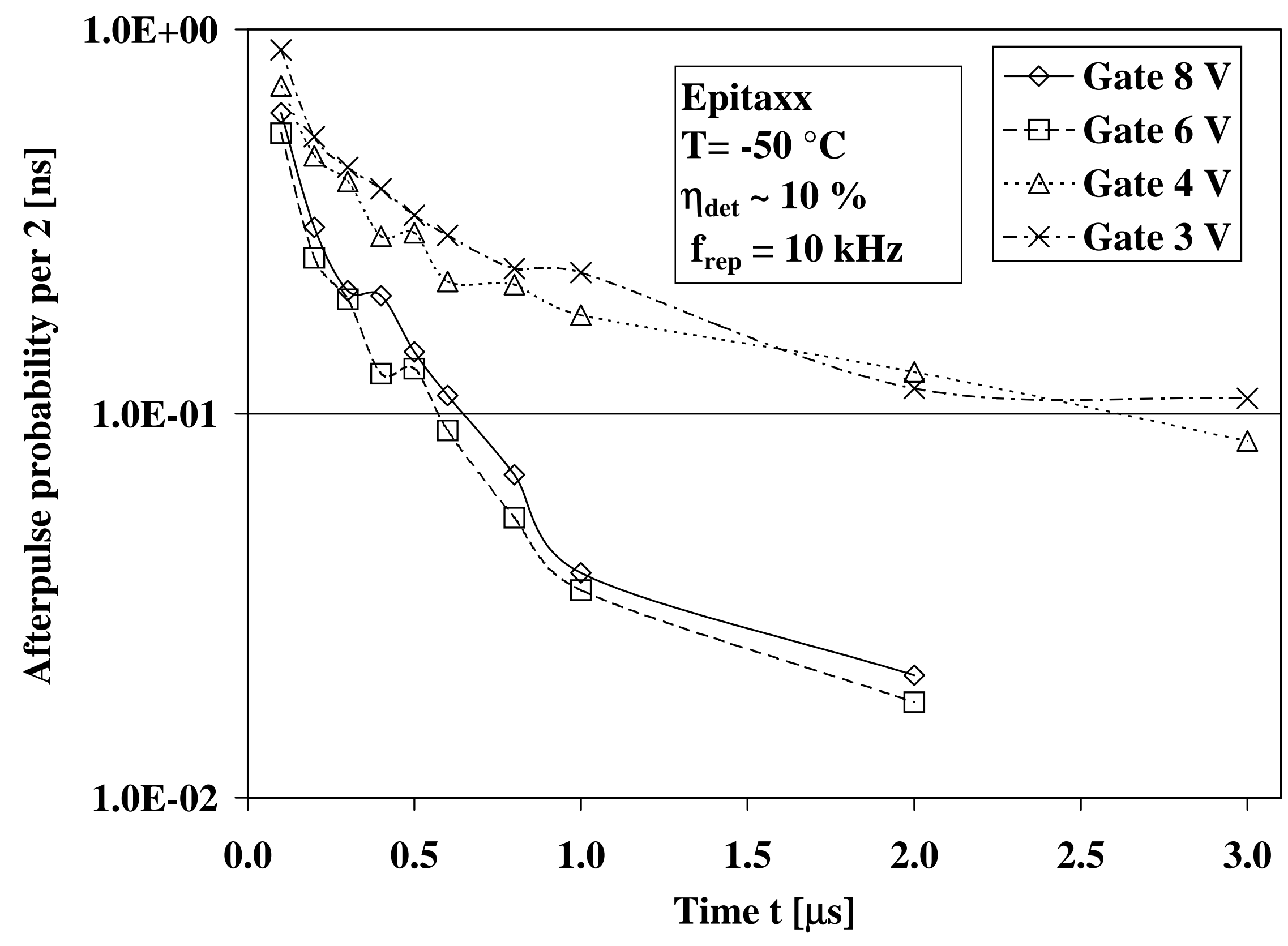


Figure 8

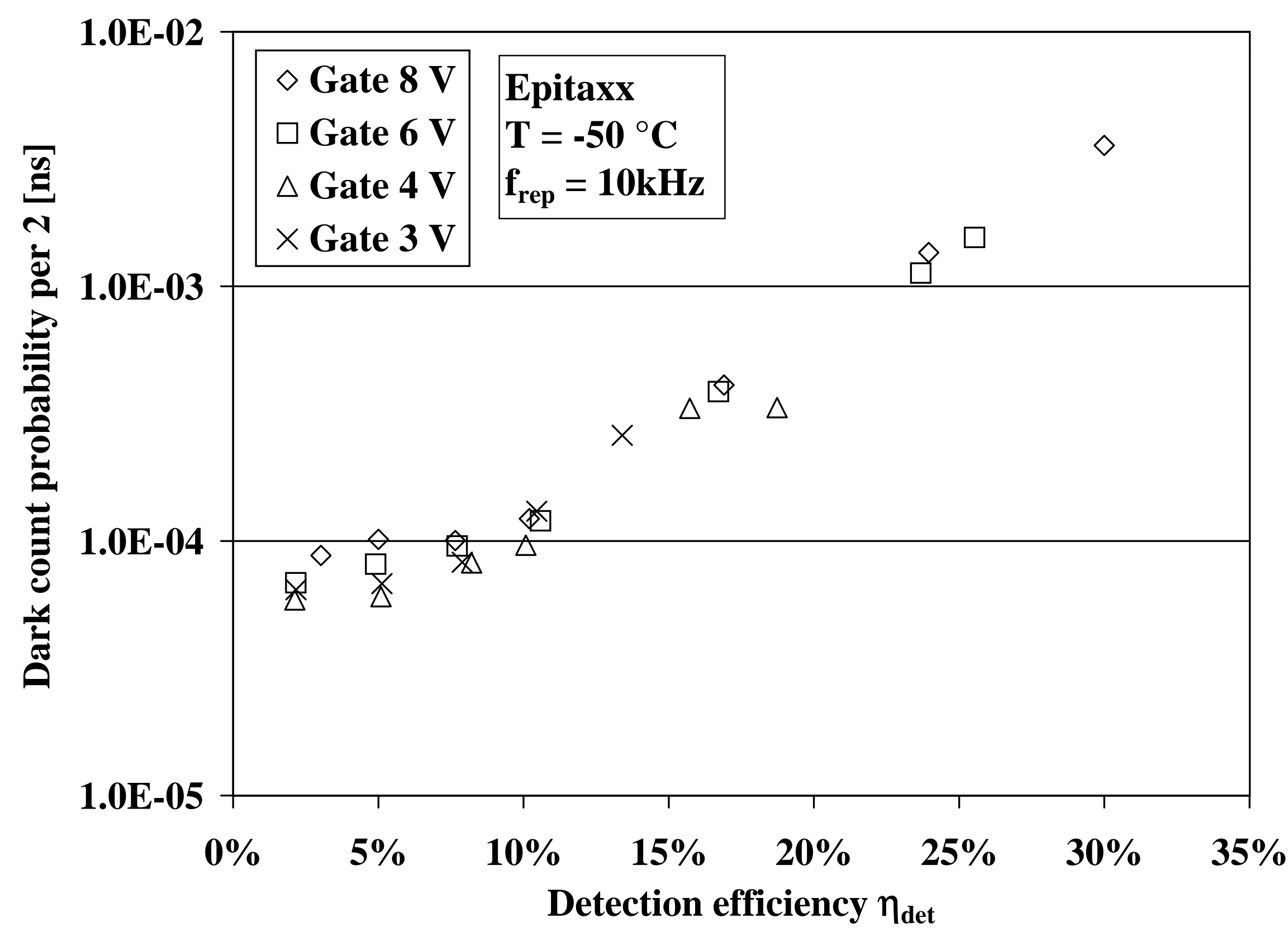


Figure 9

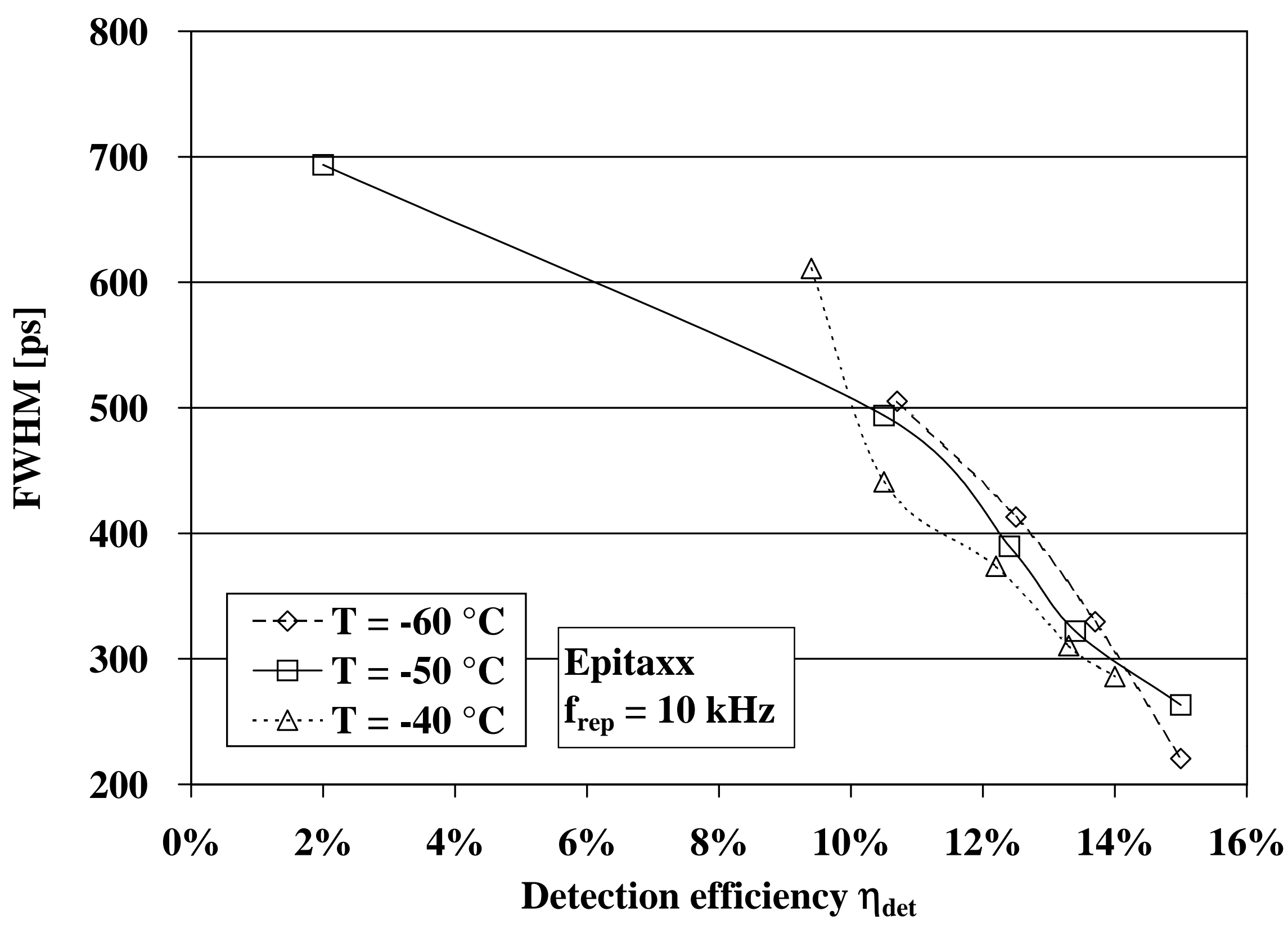


Figure 10

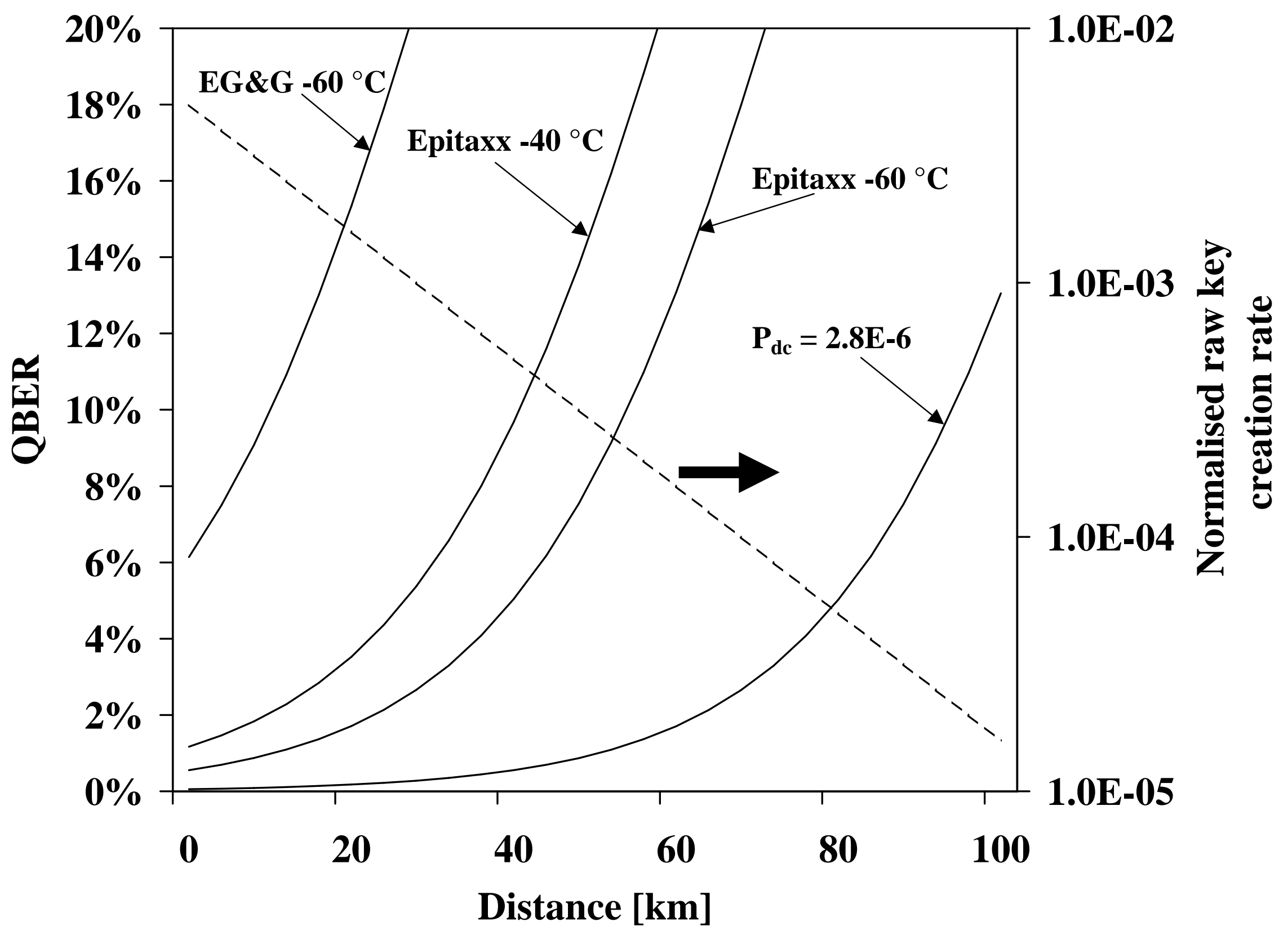

